\title{
Synthesize of a kinoform for CGH reconstruction using angler spectrum method
}

\author{
Yusei Uezono ${ }^{\mathrm{a},{ }^{*}, \text { Kouki Nakashima }}{ }^{\mathrm{b}}$, Shiyuan Yang ${ }^{\mathrm{a}}$ \\ a'Kyusyu Institute of Technology, 1-1 Sensui-cho Tobata-ku Kitakyusyu-shi Fukuoka-ken, 804-8550, \\ Japan \\ *Corresponding Author: yang@elcs.kyutech.ac.jp
}

\begin{abstract}
In this paper, we describe a Synthesize of a kinoform for $\mathrm{CGH}$ reconstruction using angler spectrum method. A hologram is a medium on which interference fringes of light are recorded. It is expected not only to represent three dimensions but also to be applied in various applications such as storage. Among them, the method of calculating by using phase is called kinoform. This method is suitable for optical reconstruction because of high light utilization efficiency
\end{abstract}

Hologram has two steps of production and reconstruction. A CGH distribution is calculated by computer. In the kinoform, since the calculation is performed with the amplitude as a fixed value, a large error occurs in the reconstructed image. In the conventional method, the error is reduced by the dummy area method, but this method causes a problem that the size of the hologram increases. The size of the hologram should not be large because it depends on the output device. In order to solve this problem, we consider using an appropriate size dummy area and iterative calculation algorithm. Therefore, we can expect to improve the resolution of the reconstructed image without changing the size of the hologram using the iterative dummy area method.

Keywords: kinoform, angler spectrum method, iterative dummy area method.

\section{Optical hologram and Computer Generated Hologram(CGH)}

\subsection{Holography}

The technique of projecting a three-dimensional image in space is called holography. Information recording the amplitude and phase information of the light emitted from the object is called a hologram. This recording and reconstruction uses light interference and diffraction. The three-dimensional image reconstructed by the hologram is the light itself emanating from the object, which can be perceived stereoscopically. When the observer moves, you can reconstruct the image from that angle.

\subsection{Optical hologram}

This chapter explains recording and reconstruction methods in optical holograms

\subsubsection{Recording}

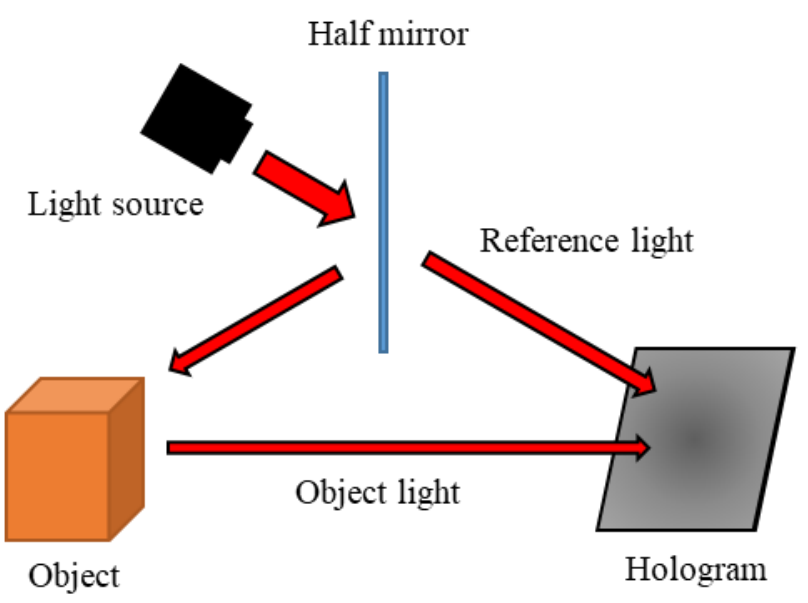

Fig. 1.1 Recording of hologram

Hologram recording uses light interference. Divide the light from the light source into two as shown in the figure. One is to irradiate the hologram as it is, the other is reflected on the object and irradiates the hologram. At this time, interference of light occurs on the hologram, and interference fringes are formed. The interference fringes 
contain phase information.

\subsubsection{Reconstruction}

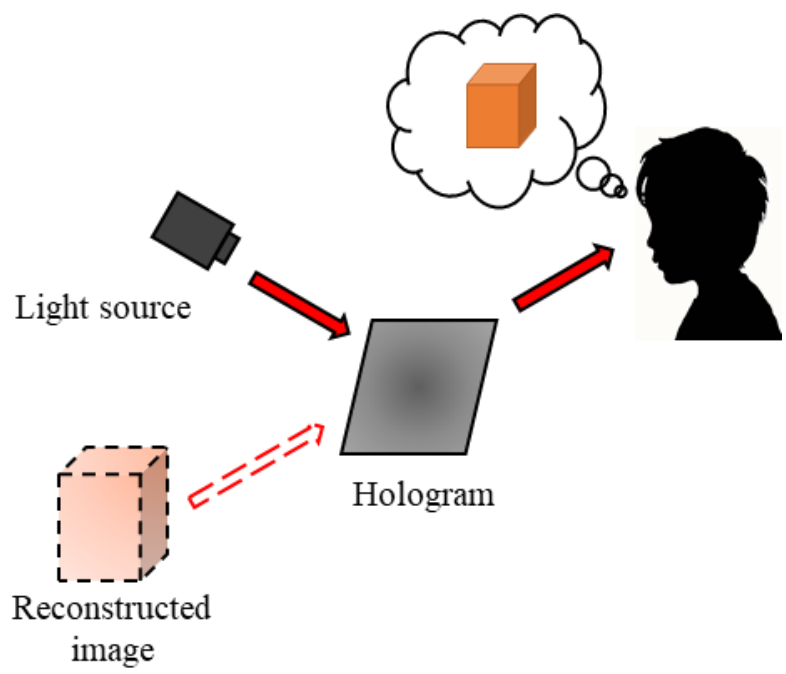

Fig. 1.2 Reconstruction of hologram

Reconstruction of hologram uses diffraction of light. By irradiating the light source in the same way as when recording the hologram, diffraction of light occurs on the hologram, and the same light as the recorded object is emitted. It is possible to observe the object at the tip of the hologram.

\subsection{Computer Generated Hologram(CGH)}

$\mathrm{CGH}$ calculates the distribution of the interference fringes of the hologram by computer by propagating light. In the optical hologram, it is a problem that the recording process requires very high precision, but in $\mathrm{CGH}$ the distribution of the hologram can be obtained only by inputting data. Also, it is possible to generate a hologram of a virtual object that does not exist.

Since CGH calculates using discrete values, it is difficult to obtain a clear reconstructed image. In this research, the purpose is to reduce the error of the reconstructed image. Also, since the computational complexity of three-dimensional recording and reconstruction becomes enormous, consideration is made using a two-dimensional image

\section{Angler spectrum method}

\section{1 angular spectrum method}

There are several methods for finding the distribution of the hologram, but in this research we use the angular spectrum method. It is an advantage that the angular spectrum method does not suffer from constraints by distance, and the calculation is fast by FFT. The angular spectral method is performed using two Fourier transformations as shown in equation (1)

$$
U(u, v)=\mathcal{F}^{-1}\left[\mathcal{F}\{g(x, y)\} \exp \left(j k z \sqrt{1-\left(\lambda f_{x}\right)^{2}-\left(\lambda f_{y}\right)^{2}}\right)\right]
$$

$$
\begin{aligned}
& g(x, y) \text { : Complex amplitude distribution of } \\
& \text { propagation source }
\end{aligned}
$$

\subsection{Recording of hologram using angler spectrum method}

When hologram recording is simulated using the angular spectrum method, two FFTs of the above equation are used. After performing the Fourier transform once, after calculating the phase delay amount in the frequency domain, band limitation is performed in order to prevent aliasing. After that, make a hoop transformation and obtain distribution of hologram

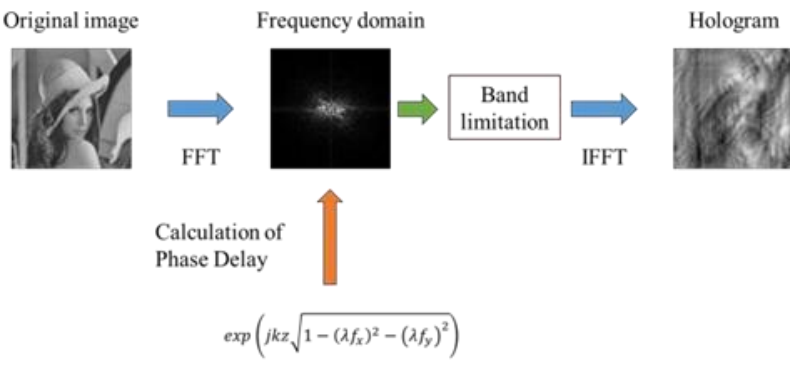

Fig. 2.1 Recording of hologram

\subsection{Reconstruction of hologram using angler spectrum method}

In order to simulate the reproduction of a hologram using the angular spectrum method, it is possible to obtain a reconstructed image by performing Fourier transformation on the hologram, calculating the phase delay amount, and 
performing Fourier transformation again.

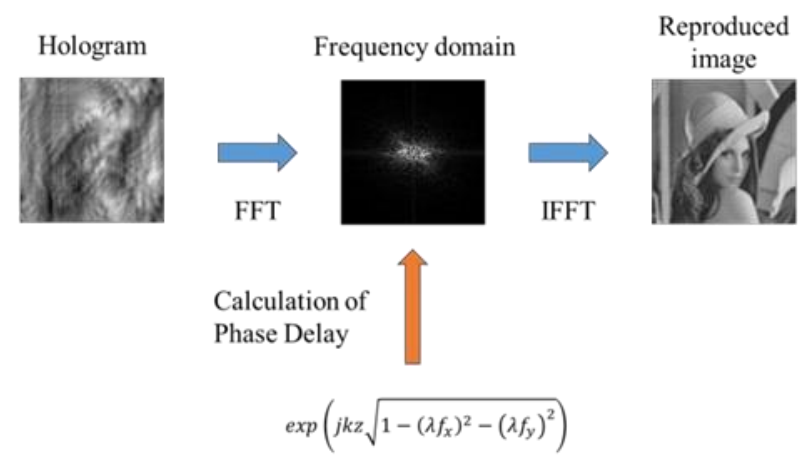

Fig. 2.2 Reconstruction of hologram

\subsection{Recording and reconstruction of hologram in kinoform}

Kinoform is a type of hologram recording phase. In the complex amplitude hologram, both the amplitude and the phase are recorded, but in the case of the kinoform, the amplitude is calculated as a constant value when the hologram is recorded. This makes the reconstructed image blurred in kinoform. Consider the method to reduce this error by the method described below.

\section{Iterative dummy area method}

\subsection{Dummy area method}

A dummy area method is one of error reduction methods in CGH. This is a method of adding a region where initial values of amplitude and phase are zero around the original image. The added region can take an arbitrary value (freedom of amplitude and phase can be used), thereby making it possible to reduce the error of the reconstructed image.

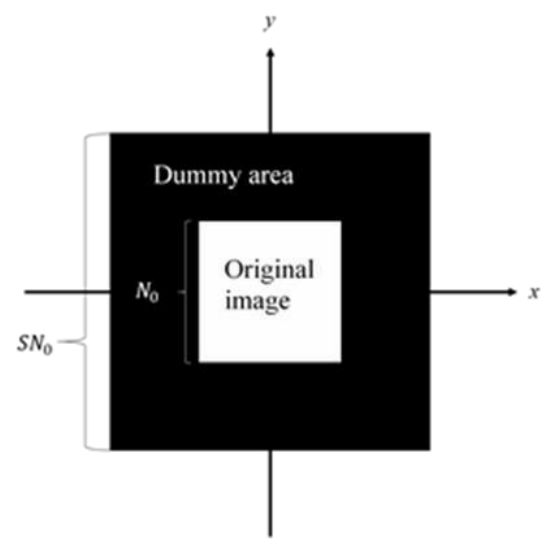

Fig. 3.1 Dummy area

\subsection{Problem of conventional dummy area method}

The dummy area method is equivalent to increasing the size of the original image. This also increases the size of the hologram. Data of the hologram is output to a highresolution printer, a spatial light modulator, or the like. The size of the hologram depends on the output device. Therefore, it is not desirable to increase the size of the hologram

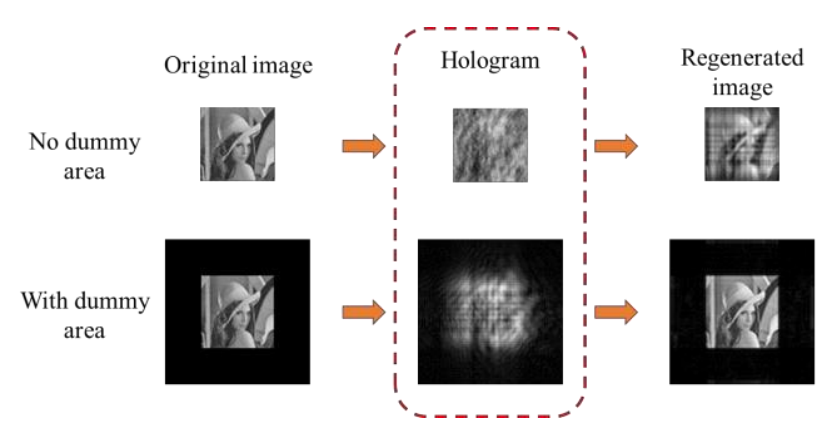

Fig. 3.2 conventional dummy area method.

\subsection{Proposed dummy area method}

In the proposed method, we stop using the whole area of the enlarged hologram for reconstruction. Cut the dummy area part of the hologram and use the hologram of the size of the original image before adding the dummy area for reconstruction.

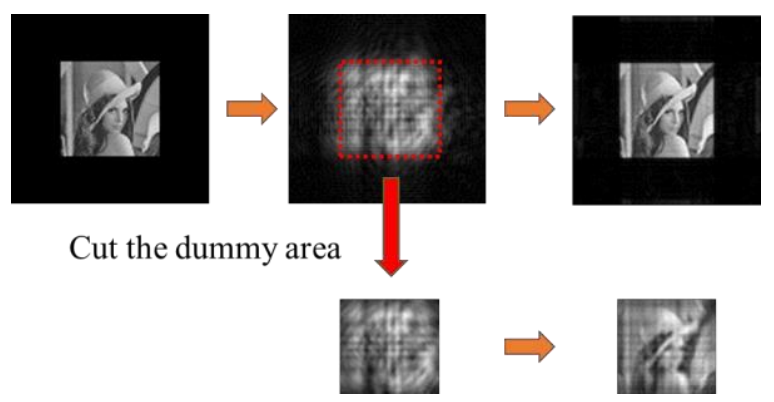

Fig. 3.3 Proposed dummy area method.

However, this method increases the error of the reconstructed image. This is because the dummy area in the hologram also contains information on the original image.

When this method is used for kinoform, large error occurs. I will explain this $\mathrm{F}$ image as an original image. If the dummy area in the hologram is cut and reproduced, a 
large error occurs

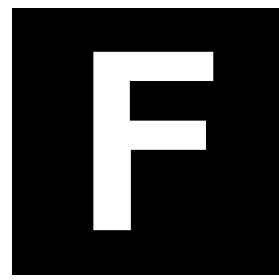

Original image

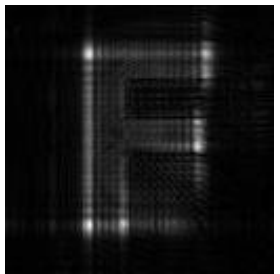

Reconstructed image 1
Fig. 3.4 reconstructed image using proposed method

Since the information of the original image is also included in the cut portion, an error occurs.

\subsection{Iterative dummy area method}

Iterative dummy area method is a method for reducing hologram error. This method is used for kinoform which reconstructs by cutting the dummy area which is the proposed method. It has been confirmed that this method can reduce errors in complex amplitude hologram using angular spectrum method.

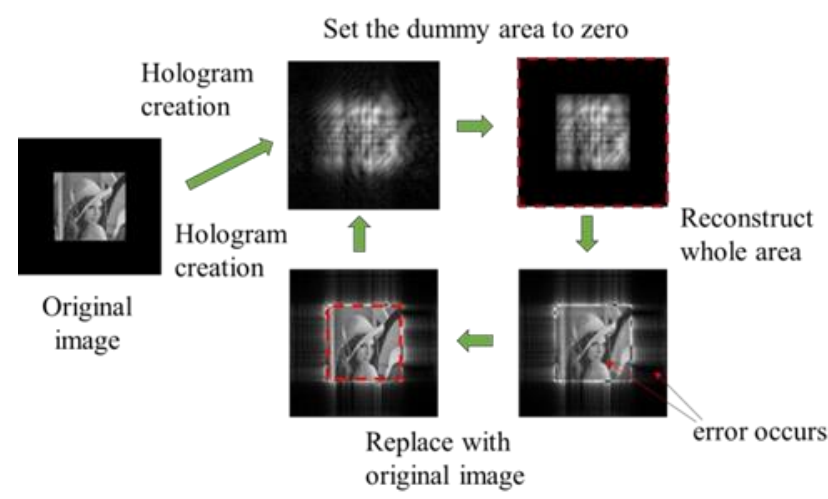

Fig. 3.5 Iterative dummy area method

As shown in Fig. 3.5, we adopt a method adding cuts of dummy areas proposed for iterative algorithm.

\section{Simulation result}

It shows the reconstructed image of the hologram recorded by the iterative algorithm using the same original image as in Figure 3.4

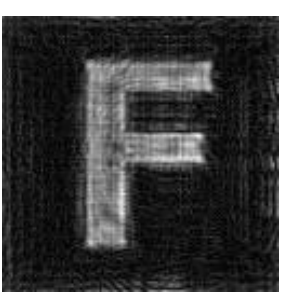

Fig. 3.5 Reconstructed image 2

The reconstructed image is the result of 1000 iterations using dummy area eight times the size of original image. In normal kinoforms, large errors occur unless calculations are performed using large size dummy areas. Next, a simulation result of a reconstructed image in the case of using twice the dummy area of the original image is shown.

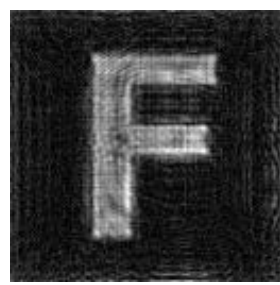

Fig. 3.6 Reconstructed image 3

It was found that there was no large difference in the reconstructed image using the dummy area of 2 times and 8 times of the original image. Such a result was also obtained when this method was used for a complex amplitude hologram.

\section{Conclusions}

We found that the proposed dummy area method in kinoform using angular spectrum method is effective. This made it possible to reduce the error without changing the size of the hologram.

\section{References}

(1) P. Harirahan, Principles of Holography, pp 1-13, pp 88- 94, (2004), Optronix Co

(2) Joseph W. Goodman, Fourier Optics (Third Edition), pp 287 - 288, pp 340 - 352, (2012), Mori Kita Publishing Co., Ltd.

(3) 